Graphical Abstract

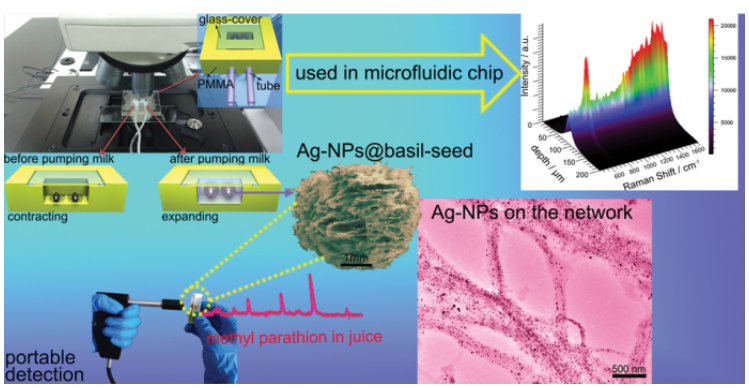

High-density plasmonic Ag nanoparticles (Ag-NPs) have been synthesized on the three-dimensional framework of natural basil-seeds as the inexpensive substrates for portable surface-enhanced Raman scattering (SERS) detection and SERS sensor. 


\title{
Dipping into a Drink: Basil-Seed Supported Silver Nanoparticles as Surface-Enhanced Raman Scattering Substrates for Toxic Molecule Detection
}

\author{
Qitao Zhou, ${ }^{a}$ Guowen Meng, ${ }^{a b *}$ Nianqiang Wu, ${ }^{c}$ Ningning Zhou, ${ }^{a}$ Bensong Chen, ${ }^{a}$ \\ Fadi Li $^{a}$ and Qing Huang ${ }^{d}$
}

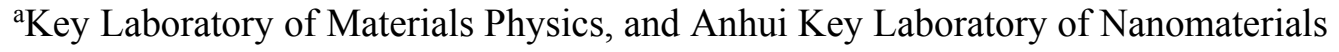
and Nanotechnology, Institute of Solid State Physics, Chinese Academy of Sciences, Hefei, 230031, China

${ }^{b}$ University of Science and Technology of China, Hefei, 230026, China

${ }^{\mathrm{c}}$ Department of Mechanical \& Aerospace Engineering, West Virginia University, P.O. Box 6106, Morgantown, WV 26506, USA

${ }^{\mathrm{d}}$ Key Laboratory of Ion Beam Bioengineering, Chinese Academy of Sciences, Hefei, 230031, China

${ }^{*}$ Corresponding author. Tel: (86) 0551-65592749, Fax: (86) 0551-65591434

E-mail: gwmeng@issp.ac.cn (G. Meng).

Keywords: basil seed, surface-enhanced Raman scattering (SERS), sensor, methyl

parathion, melamine 
ABSTRACT High-density plasmonic Ag nanoparticles (Ag-NPs) have been synthesized on a three-dimensional framework of natural basil seeds as the inexpensive substrates for surface-enhanced Raman scattering (SERS). The Ag-NPs decorated basil seeds (denoted as Ag-NPs@basil-seeds) are dipped into the analyte solution for rapid detection of methyl parathion in orange juice. Alternatively, they are incorporated into a microfluidic chip for online measurement of melamine in milk. The porous basil seeds can absorb trace melamine into the micro-cavity in the seeds, which load the melamine molecules into the gap where high-density "hot spots" appear under laser excitation. Hence the Ag-NPs@basil-seeds not only separate and pre-concentrate the trace analyte but also expose the analyte into the strong plasmonic field. This unique feature can eliminate the pre-treatment of analyte prior to SERS detection, and improve the sensitivity of the sensor. 


\section{Introduction}

Pesticides and organic additives are widely used in agriculture and food/drink production. Even trace level of pesticide residues do harm to human and ecosystem. Therefore it is essential to rapidly detect trace-level pesticides or toxic-additives in food/drink. Unfortunately it remains a challenge to realize rapid on-line detection with the existing techniques [1-5]. Surface-enhanced Raman scattering (SERS) technique has a great potential in rapid detection due to its high sensitivity, fast response and fingerprint characteristics of spectra. Various plasmonic metal nanostructure-assembled SERS-substrates have been developed for rapid detection of pesticide residues in fruits using SERS techniques. For example, Tian et al. have spread the $\mathrm{SiO}_{2}$-shell-isolated plasmonic Au-nanoparticles (Au-NPs, as active SERS-substrate) over the fresh orange contaminated by parathion as "smart dust", and realized the rapid analysis of pesticide residues by taking SERS signals in situ [6]. As for solution samples, plasmonic $\mathrm{Au} / \mathrm{Ag}$ nanostructures have been used as the active SERS-substrates for trial analysis of pesticide residues and food additives. To achieve higher SERS-activity, high density "hot spots" (sub-10-nm gaps between the $\mathrm{Au} / \mathrm{Ag}-\mathrm{NPs}$ ) are desirable, which demand high-concentration of Au/Ag colloidal NPs. Nevertheless high-concentration of NPs tends to aggregation, causing the instability problem [7-9]. To solve these problems, the carbon nanotube network with large specific surface area has been used as the framework template to load discrete $\mathrm{Au} / \mathrm{Ag}-\mathrm{NPs}$ to achieve more "hot spots" $[10,11]$. Meanwhile, biomass networks such as DNA $[12,13]$ and natural fibers $[14-16]$ have also been used as the templates. 
Although these networks exhibit excellent biocompatibility to various analytes, however, it is difficult to achieve these networked structures. On the other hand, these one-dimensional and two-dimensional structures lead to limited footprint, which makes it difficult to load a large number of plasmonic metal-NPs in a certain space. Hence three-dimensional (3D) networked SERS substrates have been developed in order to tackle this issue. However, they are usually made of large quantity of expensive porous $\mathrm{Ag} / \mathrm{Au}[17,18]$. Additional 3D SERS substrates have been fabricated by embedding Ag-NPs in sulfonated polystyrene beads [19] or by combining 3D laser photolithography with Langmuir-Blodgett nanoparticle assembly [20]. However, these synthetic methods are complicated; and it is difficult to fabricate a large area of 3D SERS substrates.

In this work, basil-seeds, a member of genus Ocimum, are used as the supporting framework to load plasmonic metal-NPs. Basil-seeds usually are used as the food additives in Asian drinks due to its medicine value and special appearance after being immersed in liquid [21, 22]. When soaked in water, basil-seed swells into a gelatinous 3D-network with polysaccharide layer expanding outwards [23]. We therefore devise a simple, in expensive, large-scale fabrication approach for decorating high-density Ag-NPs on the 3D network of basil-seeds (denoted as Ag-NPs@basil-seeds). The freeze-dried Ag-NPs@basil-seeds can be served as a protractile carrier of "hot spots" suspending in an analyte solution. Moreover, the dry contracted Ag-NPs@basil-seeds can be inserted into the micro-cavity of a microfluidic device to pump the analyte solution; and the 3D-networked SERS substrates will occupy the spare space due to 
its absorption of water and expansion. As a result, the 3D SERS substrate can be directly exposed to an incident light, circumventing the difficulty caused by analyte solution with poor transmittance (such as milk contaminated by melamine). The Ag-NPs@basil-seeds can be used as simple and inexpensive SERS-substrate, which has a promising potential in rapid detection of trace contaminants in food and environment.

\section{Experimental}

\subsection{Assembling Ag-NPs onto the 3D-networks of the basil-seeds}

$0.4 \mathrm{~g}$ of basil seeds was soaked in $100 \mathrm{~mL}$ of $\mathrm{AgNO}_{3}$ solution with different concentrations $\left(5 \times 10^{-3} \mathrm{M}, 2.5 \times 10^{-2} \mathrm{M}\right.$ and $\left.1.25 \times 10^{-1} \mathrm{M}\right)$ for $30 \mathrm{~min}$. Then the basil-seeds were filtered to remove excessive silver ions. $100 \mathrm{~mL}$ of $5 \times 10^{-2} \mathrm{M} \mathrm{NaBH}_{4}$ was added with stirring and kept for 30 min. Finally, the obtained samples were cleaned with deionized (DI) water for several times.

\subsection{Synthesis of free-standing Ag-NPs}

Following the method reported by Silvert et al [24], the as-prepared Ag-NPs were washed with ethanol several times. Finally, Ag-NPs were dispersed in DI water. The concentration was about $3 \mathrm{mg} / \mathrm{mL}$.

\subsection{Characterization}

The resulting products were characterized under a JEM-2010TEM and a field-emission SEM (Hitachi S-4800) with energy dispersive X-ray spectroscopy (EDS, Oxford), respectively. 


\subsection{SERS Measurements}

To compare the SERS activities of Ag-NPs@basil-seeds prepared under different conditions, the substrates were inserted into the SERS device respectively; and the R6G solution $\left(10^{-7} \mathrm{M}\right)$ was injected into the device. To test the SERS activity of the optimal substrate, different concentrations of R6G solutions $\left(10^{-7}, 10^{-9}\right.$ and $\left.10^{-11} \mathrm{M}\right)$ were injected into the device, respectively. The SERS spectra were recorded with a confocal microprobe Raman system (Renishaw, inVia). Before SERS detection, the substrates were soaked in the R6G solution for $10 \mathrm{~min}$. The excitation wavelength was $532 \mathrm{~nm}$ from an air-cooled argon ion laser. The swollen structures of the freeze-dried Ag-NPs@basil-seeds shortened the waiting time for absorbing water and expansion. Hence we used the freeze-dried Ag-NPs@basil-seeds for portable Raman detection. Methyl parathion contaminated juice was obtained by dissolving different amount of methyl parathion in juice. A portable Raman spectrometer (BWS415 i-Raman, BWTEK Inc.) with a $785 \mathrm{~nm}$ diode laser and $1.5 \mathrm{~m}$ lab bifurcated fiber was used for online detection. Prior to detection with a portable Raman system, the freeze-dried Ag-NPs@basil-seeds were soaked in the analyte solution for 5 min. Melamine contaminated milk was obtained by dissolving different amount of melamine in milk. In order to inject the melamine-contaminated milk into the SERS device, the shrinking Ag-NPs@basil seeds were prepared by drying under the ambient condition. Before SERS detection, the shrinking Ag-NPs@basil seeds were soaked in the injected melamine-contaminated milk for $10 \mathrm{~min}$. 


\section{Results and discussions}

\subsection{Assembling the 3D-networks of the basil-seeds with Ag-NPs and characterization}

Fig. 1 shows the fabrication process of the Ag-NPs@basil-seed SERS substrate. Briefly, basil seeds were firstly immersed in the aqueous $\mathrm{AgNO}_{3}$ solution. The $\mathrm{NaBH}_{4}$ solution was added with stirring silver ions to grow Ag-NPs on the framework of basil-seeds. Fig. S1 shows the optical photographs of both the natural dry basil seed (left) and the wet basil seed after freeze-drying (right). It can be seen that the freeze-drying of a wet basil seed generated a fluffy ball with its volume expanded dozens times. In contrast, the surface of the naturally dried basil seed was dense with wrinkles (Fig. 2a and 2b). However, the fleecy structure was formed and preserved after the basil seed was immersed in water and subsequently freeze-dried. The external 3D-network of the freeze-dried basil seed consisted of both the flakes (several hundred of microns in size) and the fibers (several hundred of nanometers in a diameter), as shown in Fig. $2 \mathrm{c}$ and $2 \mathrm{~d}$. The inherent hierarchical micro/nano structure of the natural basil seed can provide excellent framework to load metal NPs.

The size and distribution of the Ag-NPs decorated on the 3D network of the basil seeds can be tailored by tuning the $\mathrm{AgNO}_{3} / \mathrm{NaBH}_{4}$ concentration ratio (see Part $\mathrm{S} 1$ and Fig. S2 in Supporting Information). As shown in Fig. 2e-2g, when the $\mathrm{AgNO}_{3} / \mathrm{NaBH}_{4}$ concentration ratio was optimal (0.5:1 with the corresponding concentrations of $2.5 \times 10^{-2} \mathrm{M} \mathrm{AgNO}_{3}$ and $5 \times 10^{-2} \mathrm{M} \mathrm{NaBH}_{4}$, respectively), the Ag-NPs with a mean diameter of about $15 \mathrm{~nm}$ were uniformly distributed on the 
flakes (Fig. 2e-2f) and along the fiber (Fig. 2g) with very small inter-gap distance below $10 \mathrm{~nm}$, being beneficial to highly active SERS "hot spots" in the 3D network.

Energy dispersive X-ray (EDX) spectra show that the content of Ag in the product increased with the $\mathrm{AgNO}_{3}$ concentration in the preparation process (Fig. S3 in Supporting Information). The UV-Visible absorption spectra of the basil-seeds before and after decorating Ag-NPs display a new band at around $410 \mathrm{~nm}$ after being decorated with the Ag-NPs (Fig. S3b), which can be assigned to the localized surface plasmon resonance (LSPR) band of the Ag-NPs. Therefore even being soaked in water, the 3D network structure of the Ag-NPs@basil-seeds can still absorb the incident light effectively. As the swellability of the basil-seeds is recyclable, the 3D Ag-NPs@basil-seed network can be dried and easily preserved.

The Ag-NPs@basil-seeds obtained at the optimal ratio $\left(2.5 \times 10^{-2} \mathrm{M}: 5 \times 10^{-2} \mathrm{M}\right)$ showed the highest SERS activity (Fig.S4a in the Supporting Information), resulting from high-density of active "hot spots" formed in the sub-10-nm gaps among the Ag NPs dispersed on the 3D network of the basil-seeds. As shown in Fig. S4b, the resulting Ag-NPs@basil-seed with the optimal $\mathrm{AgNO}_{3} / \mathrm{NaBH}_{4}$ concentration ratio showed such a high SERS activity and allowed with the $10^{-11} \mathrm{M}$ R6G to be identified. To further examine the high SERS activity of the Ag-NPs@basil-seeds, the SERS activity of the Ag-NPs@basil-seeds was compared to that of the free-standing $35 \mathrm{~nm}$ Ag-NPs (Fig.S5a in Supporting Information). When the $3 \mathrm{mg} / \mathrm{mL}$ free-standing Ag-NPs of the SERS activity was much lower than that of the Ag-NPs@basil-seeds. Even the SERS activity of the $30 \mathrm{mg} / \mathrm{mL}$ free-standing Ag-NPs was still slightly 
weaker than that of the Ag-NPs@basil-seeds (Fig. S5b). The SERS activity of the as-prepared Ag-NPs@basil-seeds was re-checked after shelf-storage for six months. No evident change in the SERS activity of the Ag-NPs@basil-seeds was observed after six months (Fig. S6a). And no silver oxide layer was found surrounding the Ag-NP under transmission electron microscopy (TEM) (Fig.S6b). It is believed that the Ag-NPs were protected by the basil-seed network. In addition, the influence of the wavelength of incident laser was also studied (Fig. S7). The 532nm laser resulted in the best performance because it was in resonance with the SPR (band centered at 410 $\mathrm{nm})$ of the Ag-NP in the network. Although the SERS activity excited by the $785 \mathrm{~nm}$ laser was a little weaker than that by the $532 \mathrm{~nm}$ laser, it was much stronger than that by the $633 \mathrm{~nm}$ laser.

\subsection{SERS detection with the Ag-NPs@basil-seed}

The food/drink contaminants can be rapidly detected with the Ag-NPs@basil-seeds by simply immersing the freeze-dried Ag-NPs@basil-seeds into the analyte solution, followed by SERS-signal acquisition using a commercial portable Raman spectrometer (Fig.3a). No any complicated sample pretreatment steps such as extraction, preconcentration and derivatization are needed prior to detection. These advantages benefit from the unique basil seeds because their expansion upon water uptake ensures the adequate interaction between the Ag-NPs and the analyte. The swollen network can trap the analyte like tentacles with high-density "hot spots". Since the Raman spectra are recorded with a commercial portable Raman 
spectrometer, it ensures the feasibility of field-deployable detection of toxic pollutants. As shown in Fig. $3 \mathrm{~b}$ and 3c, methyl parathion dissolved in orange juice (with a concentration from $10^{-5} \mathrm{M}$ to $10^{-7} \mathrm{M}$ ) and melamine dissolved in water (with a concentration from $10^{-3} \mathrm{M}$ to $10^{-5} \mathrm{M}$ ) can be detected readily. The most pronounced bands of methyl parathion appeared at 857,1107 , and $984 \mathrm{~cm}^{-1}$ can be assigned to the in-plane deformation vibration of the triazine ring and the ring breathing Mode II, the ring breathing Mode I of the triazine ring of melamine molecule, respectively [25, 26].

On the other hand, developing compact SERS devices is an important trend for SERS-based sensing technology for real-time trace analysis [8, 27, 28]. Conventional SERS sensors with plasmonic metal nano-arrays as the SERS substrates have some limitations, such as high signal loss caused by the sealing cover of SERS device and the solution surrounding the active nanoarrays [29]. For most SERS devices, the micro-channels of the chip must be well designed to combine with the SERS substrates, making the fabrication process complicated. To overcome these limitations, the Ag-NPs@basil-seed has been integrated into a microfluidic device. As the Ag-NPs@basil-seed is on a macroscopic scale, the high requirement for microchannel design has been reduced. As shown in Fig. 4a, when the analyte solution was injected into the device embedded with the dry Ag-NPs@basil-seeds, the dry Ag-NPs@basil-seeds inside the device absorbed the aqueous analyte solution and then expanded. As a result, the analyte molecules were effectively adsorbed in the $3 \mathrm{D}$ network. The redundant solution between the Ag-NPs@basil-seeds and the upper 
glass-cover was driven away by the expanded basil-seeds and thus did not block the incident light. Since there is a glass-cover on the top, it is necessary to identify the optimal test condition. SERS mapping was implemented at different depths. As shown in Fig. 4b, the characteristic peak intensities of melamine increased when the incident light had better focus on the Ag-NPs@basil-seed. This change trend can be seen more clearly in the distribution of the band intensity at $683 \mathrm{~cm}^{-1}$ (upper right inset of Fig. 4b) and also in the corresponding depth mapping in Fig. 4c. It can be seen that the optimal depth of the focus $(90 \mu \mathrm{m})$ was almost consistent with the thickness of the upper glass-cover because the signal loss at this depth was the smallest, and the incident light had the best efficiency. The corresponding optical photo with the focus on the surface and on the depth of $90 \mu \mathrm{m}$ can be found in Fig. S9. Further SERS measurement was taken with different concentrations of melamine in milk (Fig. 5a and Table S1). A linear dependence $\left(R^{2}=0.98\right)$ was found between the intensity of the fingerprint peak $\left(683 \mathrm{~cm}^{-1}\right)$ and the logarithmic concentration of melamine (Fig.S8a in Supporting Information)

$$
y=2128.3 x+595.8
$$

where $y$ and $x$ represent the SERS signal intensity at $683 \mathrm{~cm}^{-1}$ and the logarithmic concentration of melamine, respectively. Herein the limit of detection (LOD) was defined as the concentration at which the intensity value was equal to the averaged blank intensity at $683 \mathrm{~cm}^{-1}$ plus three times the standard deviation of five blank intensities at $683 \mathrm{~cm}^{-1}[30]$. The LOD for urea melamine in milk was calculated to be $0.68 \mu \mathrm{M}$ for this sensor, which was lower than the safety limits for melamine 
ingestion, that is, $2.5 \mathrm{ppm}$ in USA and in Europe Union, and $1 \mathrm{ppm}$ for infant formula in China [31]. Most importantly, during SERS measurement, the contaminated milk was pumped into the cavity of the device directly without any pre-conditioning such as dialysis, filtration, gel filtration [32] and centrifugation [33]. The reason that the 3D Ag-NPs@basil-seed network can identify melamine in milk under the interference of proteins and fats can be explained as follows. Since melamine molecule has much smaller size and better dissolubility in water, the melamine molecules can move into the cavity much easier than the macromolecules (such as proteins or fats) by capillary attraction and diffusion during absorption of water. Furthermore, high-density "hot spots" in Ag-NPs@basil-seeds make it possible to identify the melamine signal from the SERS spectra even there are some interference signals. The SERS spectrum of pure milk and the Raman spectrum of solid melamine were compared in Fig. S8b. Clearly, the characteristic peak of melamine at $683 \mathrm{~cm}^{-1}$ did not appear in the pure milk sample.

The Raman spectra of the analyte/Ag-NPs@basil-seeds on the open space and inside the microfluidic chip were compared in Fig. 5b. Five random spots across each sample were examined under the identical experimental conditions (Fig. S10 in Supporting Information) and the average signal intensity at $683 \mathrm{~cm}^{-1}$ is shown in the inset of Fig. 5b. Due to the signal loss caused by the glass-cover, the Raman intensity taken from the microfluidic chip was about $50 \%$ of that from the open SERS substrate. 


\section{Conclusions}

In summary, the as-prepared 3D Ag-NPs@basil-seed SERS substrate has the following merits: (i) the 3D hierarchical porous framework of the basil seeds can be utilized for loading the plasmonic Ag-NPs to form a highly active 3D SERS substrate, simplifying the design and fabrication processes in the laboratory; (ii) basil seeds are abundant non-toxic natural materials at low cost; (iii) the capillary attraction to the analyte molecules accompanied by water absorption and expansion make all the preconditioning steps unnecessary prior to SERS detection. These advantages enable the Ag-NPs@basil-seed-based SERS sensor to detect methyl parathion in juice with a concentration down to $10^{-7} \mathrm{M}$. In addition, the microfluidic chip based on the Ag-NPs@basil-seeds has also been developed for rapid detection of $10^{-6} \mu \mathrm{M}$ melamine in milk, which mitigates the loss of SERS signals. In short, the Ag-NPs@basil-seeds as the 3D SERS substrates show a great potential in rapid detection of trace small molecule analytes.

\section{Acknowledgements}

We thank the National Key Basic Research Program of China (Grant 2013CB934304), the NSFC (Grant 51472245 and 11274312) and Research Supported by the CAS/SAFEA International Partnership Program for Creative Research Teams for the financial support. NW is grateful to the partially support by NSF (EPS 1003907). 


\section{References}

[1] J. Wang, L. Kong, Z. Guo, J. Xu and J. Liu, Synthesis of novel decorated one-dimensional gold nanoparticle and its application in ultrasensitive detection of insecticide, J. Mater. Chem. 20 (2010), 5271-5279.

[2] K. Ai, Y. Liu and L. Lu, Hydrogen-bonding recognition-induced color change of gold nanoparticles for visual detection of melamine in raw milk and infant formula, J. Am. Chem. Soc. 131 (2009), 9496-9497.

[3] C. Gonçalves and M. F. Alpendurada, Solid-phase micro-extraction-gas chromatography-(tandem) mass spectrometry as a tool for pesticide residue analysis in water samples at high sensitivity and selectivity with confirmation capabilities, $J$. Chromatogr. A. 1026 (2004), 239-250.

[4] H. Miao, S. Fan, Y.-N. Wu, L. Zhang, P.-P. Zhou, H.-J. Chen, Y.-F. Zhao and J.-G. Li, Simultaneous determination of melamine, ammelide, ammeline, and cyanuric acid in milk and milk products by gas chromatography-tandem mass spectrometry. Biomed, Environ. Sci. 22 (2009), 87-94.

[5] G. Huang, Z. Ouyang and R. G. Cooks, High-throughput trace melamine analysis in complex mixtures, Chem. Commun. (2009), 556-558.

[6] J. F. Li, Y. F. Huang, Y. Ding, Z. L. Yang, S. B. Li, X. S. Zhou, F. R. Fan, W. Zhang, Z. Y. Zhou, D. Y. Wu, B. Ren, Z. L. Wang and Z. Q. Tian, Shell-isolated nanoparticle-enhanced Raman spectroscopy. Nature, 464 (2010), 392-395. 
[7] B. L. Darby and E. C. Le Ru, Competition between molecular adsorption and diffusion: Dramatic consequences for SERS in colloidal solutions, J. Am. Chem. Soc. 136 (2014), 10965-10973.

[8] B. D. Piorek, S. J. Lee, J. G. Santiago, M. Moskovits, S. Banerjee and C. D. Meinhart, Free-surface microfluidic control of surface-enhanced Raman spectroscopy for the optimized detection of airborne molecules. Proc. Natl. Acad. Sci. USA. 104 (2007), 18898-18901.

[9] D. Lee, S. Lee, G. H. Seong, J. Choo, E. K. Lee, D.-G. Gweon and S. Lee, Quantitative analysis of methyl parathion pesticides in a polydimethylsiloxane microfluidic channel using confocal surface-enhanced Raman spectroscopy, Appl. Spectrosc. 60 (2006), 373-377.

[10] S. Lee, M. G. Hahm, R. Vajtai, D. P. Hashim, T. Thurakitseree, A. C. Chipara, P. M. Ajayan and J. H. Hafner, Utilizing 3d SERS active volumes in aligned carbon nanotube scaffold substrates. Adv. Mater. 24 (2012), 5261-5266.

[11] Y. H. Sun, K. Liu, J. Miao, Z. Y. Wang, B. Z. Tian, L. N. Zhang, Q. Q. Li, S. S. Fan and K. L. Jiang, Highly sensitive surface-enhanced Raman scattering substrate made from superaligned carbon nanotubes, Nano Lett. 10 (2010), 1747-1753.

[12] G. Wei, L. Wang, Z. Liu, Y. Song, L. Sun, T. Yang and Z. Li, DNA-network-templated self-assembly of silver nanoparticles and their application in surface-enhanced Raman scattering, J. Phys. Chem. B. 109 (2005), 23941-23947. 
[13] L.-b. Yang, G.-y. Chen, J. Wang, T.-t. Wang, M.-q. Li and J.-h. Liu, Sunlight-induced formation of silver-gold bimetallic nanostructures on DNA template for highly active surface enhanced Raman scattering substrates and application in TNT/tumor marker detection, J. Mater. Chem. 19 (2009), 6849-6856.

[14] M.-L. Cheng, B.-C. Tsai and J. Yang, Cheng, M. L.; Tsai, B. C.; Yang, J. Silver nanoparticle-treated filter paper as a highly sensitive surface-enhanced Raman scattering (SERS) substrate for detection of tyrosine in aqueous solution, Anal. Chim. Acta. 708 (2011), 89-96.

[15] Y. H. Ngo, D. Li, G. P. Simon and G. Garnier, Gold nanoparticle-paper as a three-dimensional surface enhanced Raman scattering substrate. Langmuir. 28 (2012), 8782-8790.

[16] C. H. Lee, L. Tian and S. Singamaneni, Paper-based SERS swab for rapid trace detection on real-world surfaces, ACS Appl. Mater. Inter. 2 (2010), 3429-3435.

[17] G. Li, X. Song, Z. Sun, S. Yang, B. Ding, S. Yang, Z. Yang and F. Wang, Nanoporous Ag prepared from the melt-spun Cu-Ag alloys, Solid State Sci. 13 (2011), $1379-1384$.

[18] Y. X. Zhang and H. C. Zeng, Gold sponges prepared via hydrothermally activated self-assembly of Au nanoparticles, J. Phys. Chem. C. 111 (2007), 6970-6975. 
[19] JB.-H. Jun, J.-H. Kim, H. Park, J.-S. Kim, K.-N. Yu, S.-M. Lee, H. Choi, S.-Y. Kwak, Y.-K. Kim, D. H. Jeong, M.-H. Cho and Y.-S. Lee. Surface-enhanced Raman spectroscopic-encoded beads for multiplex immunoassay. J. Comb. Chem. 9 (2007), 237-244.

[20] Q. Zhang, Y. H. Lee, I. Y. Phang, C. K. Lee and X. Y. Ling. Hierarchical 3D SERS substrates fabricated by integrating photolithographic microstructures and self-assembly of silver nanoparticles. Small 10 (2014), 2703-2711.

[21] R. J. Abbas, Effect of using fenugreek, parsley and sweet basil seeds as feed additives on the performance of broiler chickens, Int. J. Poult. Sci. 9 (2010), 278-282.

[22] S. Razavi, A. Bostan and M. Rezaie, Image processing and physic-mechanical properties of basil seed (ocimum basilicum), J. Food Process Eng. 33 (2010), 51-64.

[23] J.-i. Azuma and M. Sakamoto, Cellulosic hydrocolloid system present in seed of plants, Trends Glycosci. Glycotechnol. 15 (2003), 1-14.

[24] K. TekaiaáElhsissen, Preparation of colloidal silver dispersions by the polyol process. Part 1-synthesis and characterization, J. Mater. Chem. 6 (1996), 573-577.

[25] L. He, Y. Liu, M. Lin, J. Awika, D. R. Ledoux, H. Li and A. Mustapha, A new approach to measure melamine, cyanuric acid, and melamine cyanurate using surface enhanced Raman spectroscopy coupled with gold nanosubstrates, Sens. Instrum. Food Qual. Saf. 2 (2008), 66-71. 
[26] E. Kämmer, T. Dörfer, A. Csaki, W. Schumacher, P. A. Da Costa Filho, N. Tarcea, W. Fritsche, P. Roesch, M. Schmitt and J. Popp, Evaluation of colloids and activation agents for determination of melamine using UV-SERS, J. Phys. Chem. C. 116 (2012), 6083-6091.

[27] L. X. Quang, C. Lim, G. H. Seong, J. Choo, K. J. Do and S.-K. Yoo, A portable surface-enhanced Raman scattering sensor integrated with a lab-on-a-chip for field analysis, Lab Chip. 8 (2008), 2214-2219.

[28] K. R. Strehle, D. Cialla, P. Rösch, T. Henkel, M. Köhler and J. Popp, A reproducible surface-enhanced Raman spectroscopy approach. Online SERS measurements in a segmented microfluidic system, Anal. Chem. 79 (2007), $1542-1547$.

[29] H. Mao, W. Wu, D. She, G. Sun, P. Lv and J. Xu, Microfluidic surface-enhanced Raman scattering sensors based on nanopillar forests realized by an oxygen-plasma-stripping-of-photoresist technique, Small. 10 (2014), 127-134.

[30] H.-Y. Wu, C. J. Choi and B. T. Cunningham. Plasmonic nanogap-enhanced Raman scattering using a resonant nanodome array. Small 8 (2012), 2878-2885.

[31] L. Zhu, G. Gamez, H. Chen, K. Chingin and R. Zenobi, Rapid detection of melamine in untreated milk and wheat gluten by ultrasound-assisted extractive electrospray ionization mass spectrometry (EESI-MS), Chem. Commun. (2009), $559-561$. 
[32] A. Kim, S. J. Barcelo, R. S. Williams and Z. Li, Melamine sensing in milk products by using surface enhanced Raman scattering, Anal. Chem. 84 (2012), 9303-9309.

[33] X. F. Zhang, M. Q. Zou, X. H. Qi, F. Liu, X. H. Zhu and B. H. Zhao, Detection of melamine in liquid milk using surface-enhanced Raman scattering spectroscopy, $J$. Raman Spectrosc. 41 (2010), 1655-1660. 


\section{Figure captions.}

Fig. 1 Schematic of the fabrication of 3D Ag-NPs@basil-seed network. The lower insets are optical photos of the products.

Fig. 2 (a) and (b) SEM images of the dry basil-seed and the close-up view. (c) and (d) SEM images of the soaked basil-seed after freeze-drying and the close-up view. (e) and (f) TEM images of Ag-NPs decorated on the flakes of the 3D network prepared with the concentration ratio of $\mathrm{AgNO}_{3}$ and $\mathrm{NaBH}_{4}$ : $0.5: 1$ and the corresponding close-up view. (g) TEM images of Ag-NPs decorated on the fiber of the 3D network prepared with the concentration ratio of $\mathrm{AgNO}_{3}$ and $\mathrm{NaBH}_{4}: 0.5: 1$. The inset is the corresponding particle size histogram.

Fig. 3 (a) The optical photo of the portable Raman system and schematic of the measurement. (b) Detection of methyl parathion in orange juice. (c) Detection of melamine in water.

Fig. 4 (a) Schematic illustration of Raman system combined with the microfluidic SERS-sensor (inset the functional seeds as SERS substrates). (b) Variation in SERS gain under monotonic tuning of the focus depth of the exciting light. The upper right inset is the distribution of the band intensity at $683 \mathrm{~cm}^{-1}$. (c) The corresponding depth SERS mapping.

Fig. 5 (a) Detection of different concentrations of melamine in milk with the focus depth of $90 \mu \mathrm{m}$. (b) Raman spectra of the same analyte measured in the microfluidic SERS-sensor and on the open space. The upper inset is the schematic illustration of the difference between our sensor and a conventional sensor. The inset histogram is the comparison of the average signal intensity at $683 \mathrm{~cm}^{-1}$ of the same analyte measured in the microfluidic SERS-sensor and on the open space. 
Immersing in $\mathrm{AgNO}_{3}$ solution
Adding $\mathrm{NaBH}_{4}$ solution
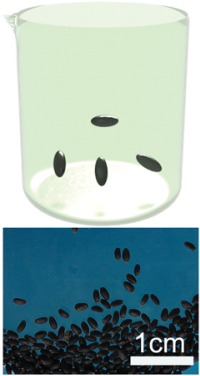
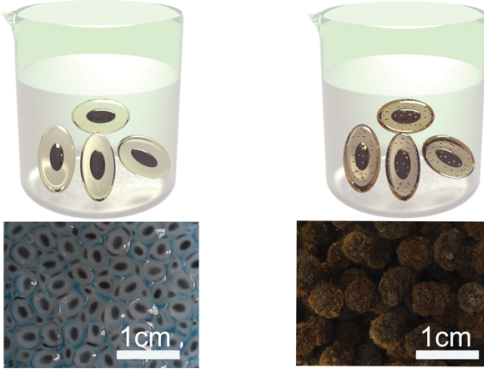
Figure 2
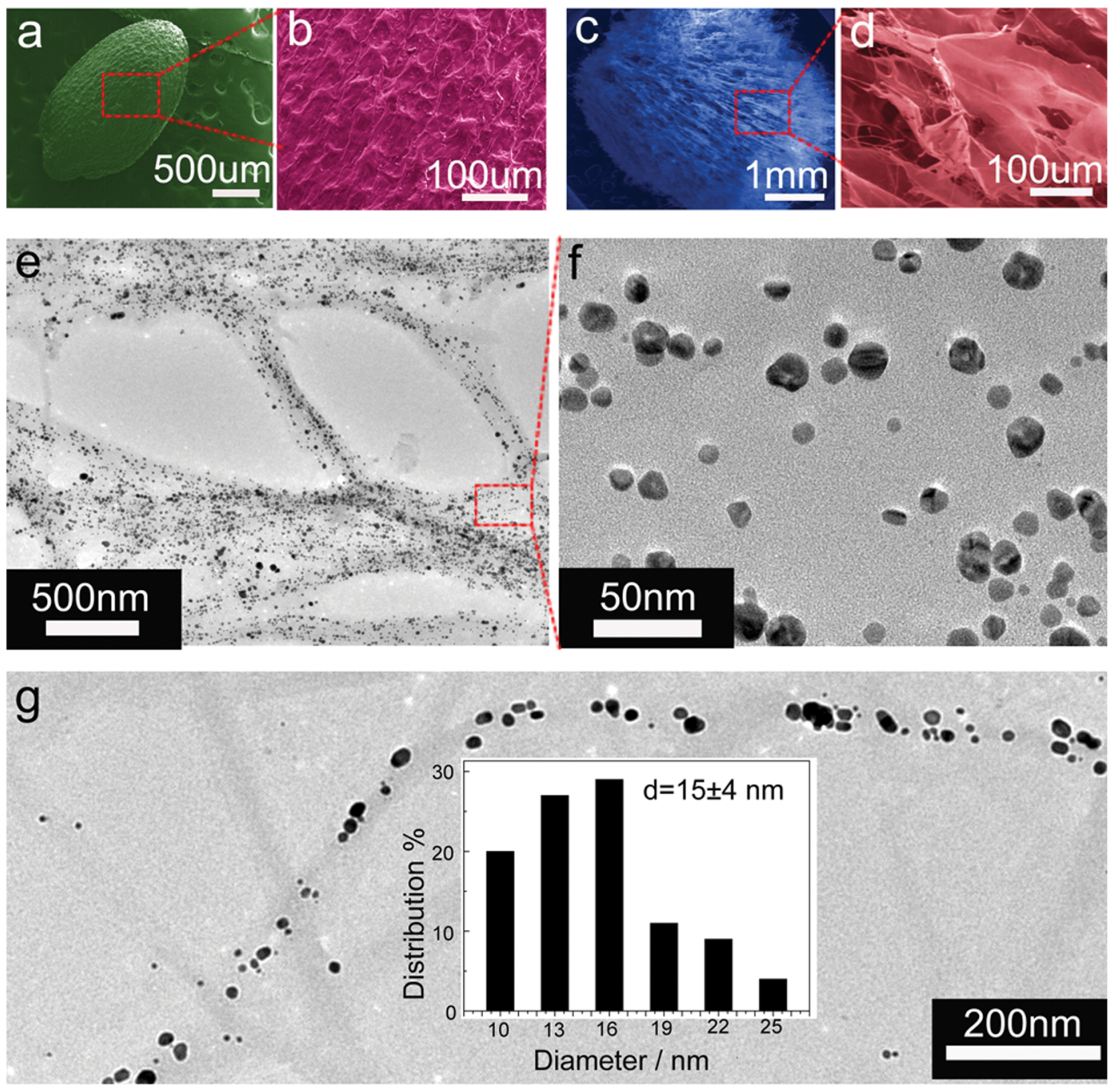

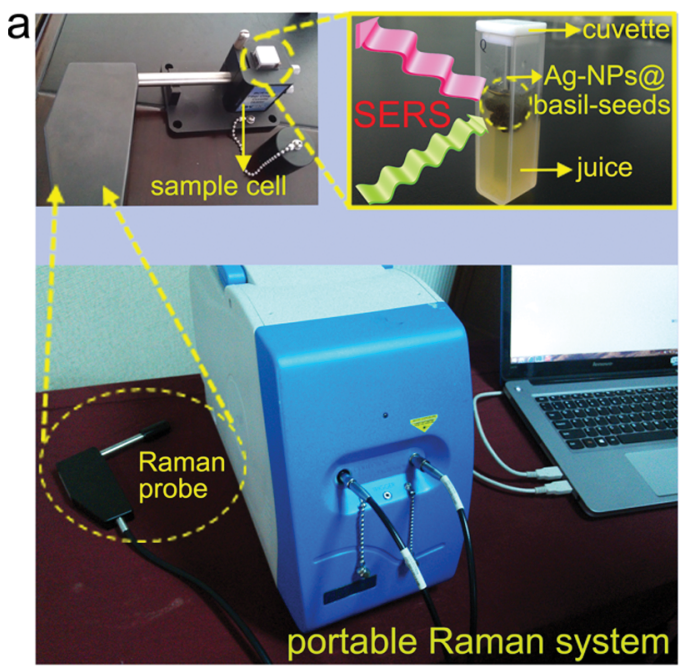

b methyl parathion juice solution

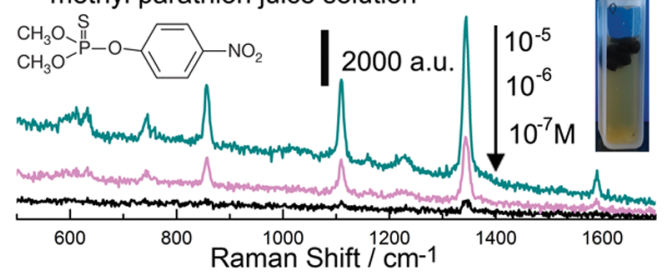

C melamine water solution

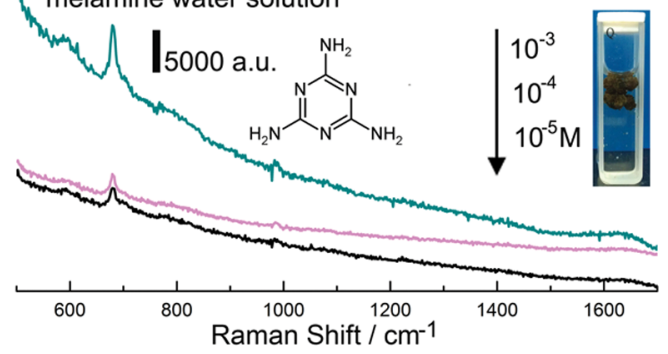



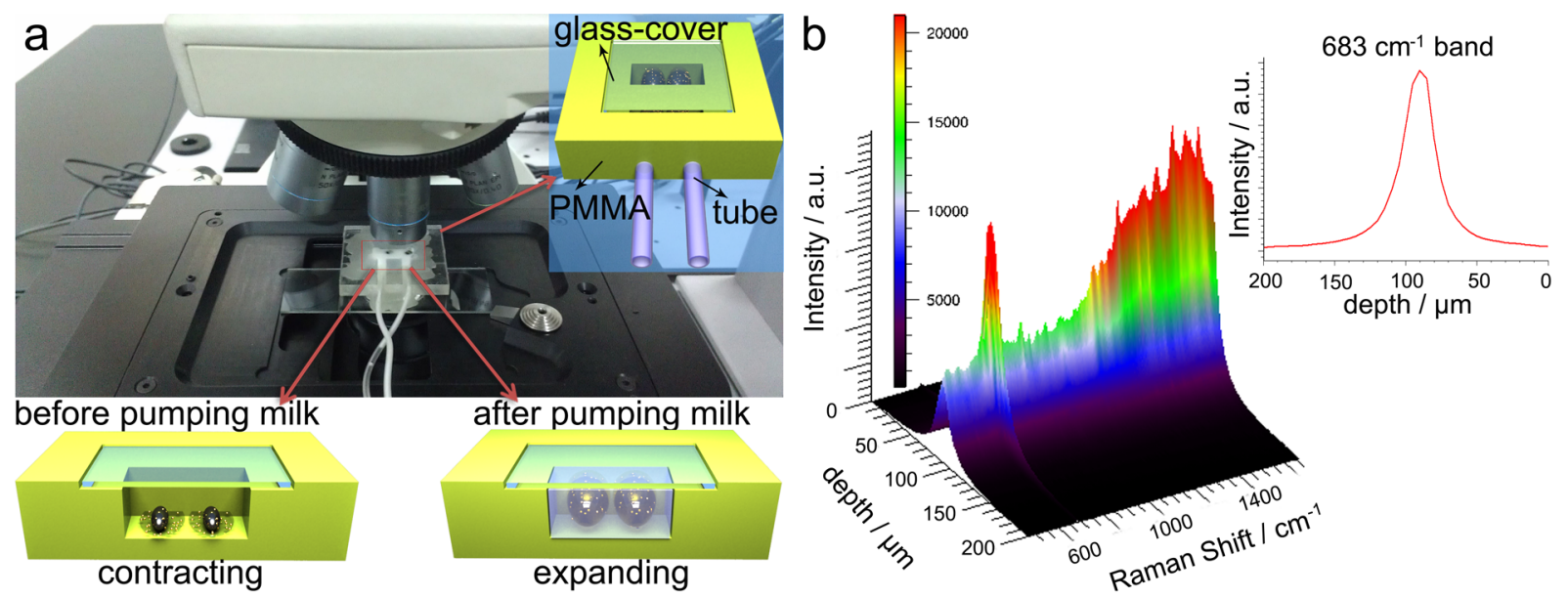

C

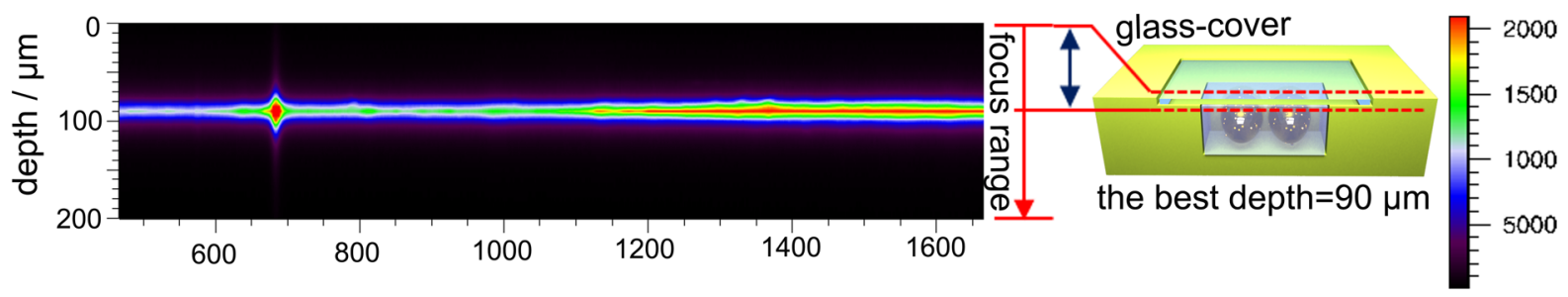

Raman Shift $/ \mathrm{cm}^{-1}$ 


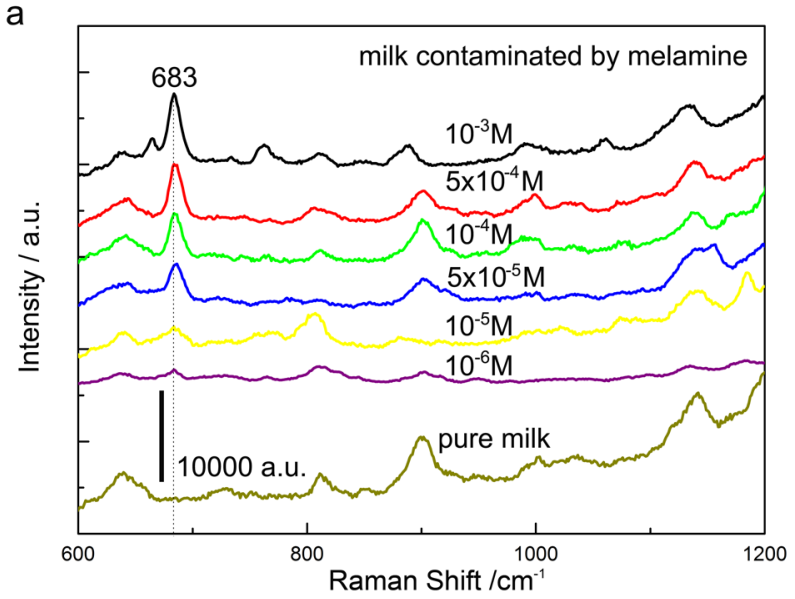

b normal SERS sensor

\section{weakened by} glass-cover and solution

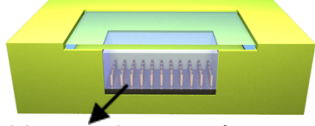

noble metal nanorod arrays our SERS sensor avoid being weakened by solution

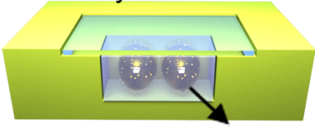

Ag-NPs@basil-seed

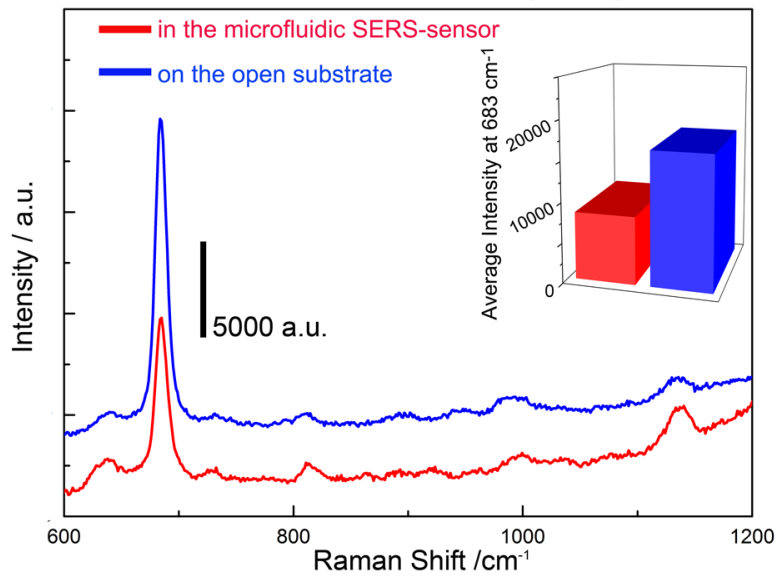


Qitao Zhou is a student in Institute of Solid State Physics (ISSP), Chinese Academy of Sciences (CAS), and he received his PhD degree in Material Physics and Chemistry from University of CAS in 2015. His research interests include nanomaterials and surface-enhanced Raman scattering (SERS) sensors.

Guowen Meng has worked as a professor in ISSP, CAS, since 1999. He received his PhD degree in Materials Science and Engineering from Northwestern Polytechnic University, Xi'an, in 1996. His current research focuses on 1D nanomaterials and their sensitivity to pollutants.

Dr. Nianqiang (Nick) Wu is a professor in the Department of Mechanical \& Aerospace Engineering at West Virginia University, USA. He received his Ph.D. degree from Zhejiang University, China in 1997. He was a Postdoctoral Research Fellow at the University of Pittsburgh from 1999 to 2001. He managed the Keck Interdisciplinary Surface Science Center at Northwestern University in USA from 2001 to 2005. He has been holding a faculty position at West Virginia University since 2005. His current research interests include nanomaterials and nanolithography, photocatalysts and photoelectrochemical cells, chemical sensors, biosensors and lab-on-chips.

Ningning Zhou is a student in Institute of Solid State Physics (ISSP), Chinese Academy of Sciences (CAS), and she will receive her PhD degree in Material Physics and Chemistry from University of CAS in 2017. Her research interests include nanomaterials and surface-enhanced Raman scattering (SERS) sensors.

Bensong Chen is an assistant professor in Institute of Solid State Physics (ISSP), Chinese Academy of Sciences (CAS), and he received his $\mathrm{PhD}$ degree in Materials Science from University of CAS in 2010. His research interests include nanomaterials and surface-enhanced Raman scattering (SERS) sensors.

Fadi Li is an assistant engineer in Institute of Solid State Physics (ISSP), Chinese Academy of Sciences (CAS), and he received his master's degree in Optical Engineering from Shenzhen University in 2011. His research interests include Raman spectroscopy.

Qing Huang is a professor working at Hefei Institutes of Physical Science, CAS. He obtained his Ph.D degree from Goettingen University, Germany in 2000. His research interests include spectroscopy applications in physics, chemistry, and biology. 